\title{
BMJ Open Comparative effect of nutraceuticals on lipid profile: a protocol for systematic review and network meta-analysis
}

\author{
Jakub Morze (D) , ${ }^{1}$ Tadeusz Osadnik, ${ }^{2,3}$ Kamila Osadnik, ${ }^{2}$ Mateusz Lejawa, ${ }^{2}$ \\ Grzegorz Jakubiak, ${ }^{2}$ Natalia Pawlas (D) ,2 Mariusz Gasior, ${ }^{4}$ Lukas Schwingshackl, ${ }^{5}$ \\ Maciej Banach ${ }^{6,7,8}$
}

To cite: Morze J, Osadnik T, Osadnik K, et al. Comparative effect of nutraceuticals on lipid profile: a protocol for systematic review and network meta-analysis. BMJ Open 2020;10:e032755. doi:10.1136/ bmjopen-2019-032755

- Prepublication history and additional material for this paper are available online. To view these files, please visit the journal online (http://dx.doi. org/10.1136/bmjopen-2019032755).

Received 03 July 2019 Revised 21 May 2020 Accepted 03 July 2020

Check for updates

(C) Author(s) (or their employer(s)) 2020. Re-use permitted under CC BY-NC. No commercial re-use. See rights and permissions. Published by BMJ.

For numbered affiliations see end of article.

Correspondence to

Jakub Morze;

jakub.morze@uwm.edu.pl

\section{ABSTRACT}

Introduction According to the common definition, nutraceuticals are components found in food that can act as therapeutic substances. Recently, the International Lipid Expert Panel published two position papers covering the topic of lipid-lowering nutraceuticals and their potential use as a complementary treatment in addition to statins or as an alternative treatment in statin-intolerant patients. The aim of this study was to compare the effect of different nutraceuticals on lipid profiles in a systematic review with pairwise and network meta-analyses. Methods and analysis Three databases, including PubMed, Embase and the Cochrane Central Register of Controlled Trials, will be searched without time or publication language restrictions. The estimated end date for the searches will be 29 March 2020. Each stage of the review, including the study section, data extraction, and risk of bias and quality of evidence assessments, will be performed in duplicate. Randomised controlled trials meeting the following criteria will be eligible for inclusion: (1) participants aged $\geq 18$ years, (2) intervention with a selected nutraceutical (artichoke, berberine, bergamot, soluble fibres, green tea, garlic, lupin, plant sterols and stanols, red yeast rice, soybean, spirulina or a combination of the aforementioned nutraceuticals), (3) administration of the treatment in the form of capsules, pills, powders, solutions, tablets or enriched food items, (4) comparison with another nutraceutical or placebo, (5) intervention period $\geq 3$ weeks and (6) lipid profile (low-density lipoprotein cholesterol, high-density lipoprotein cholesterol, total cholesterol, triglycerides) as an outcome. Randomeffect pairwise and network meta-analyses will be used to summarise the relative effect of each nutraceutical in comparison to the effect of every other nutraceutical. Subgroup analyses will be stratified by age, sex, ethnicity, sample size, length of trial follow-up, baseline cholesterol level and presence of other comorbidities.

Ethics and dissemination This review will summarise findings from primary studies, and therefore no ethics approval is required. The results will be presented at conferences as well as published in a peer-reviewed journal.

PROSPERO registration number CRD42019132877.

\section{Strengths and limitations of this study}

- This network meta-analysis will be the first to explore the comparative effectiveness of different nutraceuticals on lipid profile.

$\checkmark$ Results of our review will address the question which nutraceutical is the most promising in terms of lowering plasma lipids.

- Variations in trial design, base patient characteristics, doses and administration mode of nutraceuticals, which may increase heterogeneity and inconsistency of networks, will be addressed by appropriate subgroup analyses.

- Different mechanisms of action of various nutraceuticals.

- Low number of eligible head-to-head trials may produce sparsely connected network of treatments.

\section{INTRODUCTION}

Cardiovascular diseases (CVDs) account for one-third of deaths and remain as the most common cause of mortality worldwide. ${ }^{1}$ According to the Global Burden of Diseases, in 2015, the estimated global prevalence of CVD was more than 420 million cases. ${ }^{2}$ Among many modifiable risk factors, such as smoking cessation, changes in dietary habits, weight loss, and blood pressure and glucose control, lowering plasma cholesterol remains a key factor in the primary prevention of CVD. ${ }^{3}$

Statins remain the most widely used lipidlowering drugs for CVD prevention. ${ }^{4} \mathrm{~A}$ large body of evidence from high-quality randomised clinical trials suggests that statin therapy is effective at reducing levels of serum low-density lipoprotein cholesterol (LDL-C) and total cholesterol (TC) as well as the risk of cardiovascular events and deaths. ${ }^{5}$ The current American College of Cardiology/American Heart Association guidelines recommend the inclusion of statins for primary prevention in patients with diabetes, 
severe hypercholesterolemia and mild-to-moderate hypercholesterolemia depending on individual CVD risk. ${ }^{6}$

Despite the good general safety profile of statins, recent concerns have been raised about the prevalence of statinassociated muscle syndrome or transient elevation of liver syndromes and other less frequent adverse effects like occurrence of diabetes mellitus. ${ }^{7}$ Second-line drugs like ezetimibe, although less frequently, can also cause muscle pain and liver enzyme elevation. ${ }^{8}$ Drugs like bile acid sequestrants or niacin are rarely used mainly due to adverse effects leading to non-compliance. ${ }^{9}{ }^{10}$ Discontinuation of lipid-lowering treatment is associated with an increased risk of atherosclerotic CVD and mortality. ${ }^{11}$ Moreover, prescription of lipid-lowering drugs for the elderly patients in primary prevention is a subject of debate. ${ }^{12}$ These issues have shifted efforts to identifying evidence for alternative therapies, whose use was not extensively addressed in previous guidelines.

Due to lipid-lowering potential, some nutraceuticals can be considered as an option in the therapy of lipid disorders. According to common definitions, nutraceuticals are components found in food that can act as therapeutic substances. ${ }^{13}$ The potential lipid-lowering activity of selected nutraceuticals can be explained by inhibiting liver cholesterol synthesis, decreasing cholesterol absorption and increasing cholesterol excretion, as well as influencing fatty acid metabolism. ${ }^{14}$ Additional benefits from the use of nutraceuticals have been demonstrated on glycaemic control, blood pressure, endothelial function and subclinical inflammation. ${ }^{15} 16$ A large body of systematic reviews and meta-analyses reported a beneficial effect of different nutraceuticals on plasma LDL-C, high-density lipoprotein cholesterol (HDL-C), triglycerides (TG) and TC. ${ }^{17-23}$ However, the vast majority of these reviews did not report the quality of the summarised evidence, which is a key issue to address the confidence in the obtained results and inform clinical guidelines.

The International Lipid Expert Panel has published two position papers covering the topic of lipid-lowering nutraceuticals and their potential use as a complementary treatment to statins or an alternative therapy for patients with statin intolerance. ${ }^{24}$ Since both reports described the efficiency and safety of single or combined nutraceuticals in comparison with placebo or a control treatment, no conclusions were made in terms of the superiority or noninferiority of using certain nutraceuticals over others. We want to extend the findings of these two position papers to address these issues by simultaneously comparing different nutraceuticals.

Therefore, our aim is to compare the effect of different nutraceuticals on lipid profiles in a systematic review with pairwise and network meta-analyses.

\section{METHODS AND ANALYSIS}

The protocol for this systematic review and network meta-analysis was registered in the PROSPERO International Prospective Register of Systematic Reviews. The methods for conducting and describing the results of this review have been planned according to Preferred Reporting Items for Systematic Reviews and MetaAnalyses (PRISMA) and its extensions dedicated to network meta-analyses. ${ }^{25} 26$ The text of the protocol was written according to the Preferred Reporting Items for Systematic Review and Meta-Analysis Protocols guidelines for reporting protocols. ${ }^{27}$

\section{Patient and public involvement}

Patients and the public were not involved in the design or planning of the study.

\section{Eligibility criteria}

Studies fulfilling the eligibility criteria listed below will be included in the systematic review.

\section{Participants}

We will only include studies conducted with adults (subjects aged $\geq 18$ years). Since previous meta-analyses indicated the vast use of nutraceuticals in patients with other metabolic disorders associated with increased CVD risk, participants will be included regardless of disease, normocholesterolemic or hypercholesterolemic status. ${ }^{17-23}$ However, these conditions will provide a basis for appropriate subgroup analyses.

\section{Interventions and comparators}

The literature includes data on more than 40 single and combined lipid-lowering nutraceuticals. ${ }^{28}$ Since LDL-C is a primary lipid target in CVD prevention, ${ }^{3}$ the scope of this review will be limited to nutraceuticals with the potential to lower LDL-C. To identify eligible agents, we carefully evaluated recent position papers on nutraceuticals from the International Lipid Expert Panel and related meta-analyses. ${ }^{14}{ }^{24}$ The following interventions will be considered for inclusion:

- Artichoke-extracts from Cynara scolymus and C. cardunculus.

- Berberine-isoquinoline alkaloids isolated from barberry (Berberis vulgaris), tree turmeric (B. aristata), goldenseal (Hydrastis canadensis) or Oregon grape (B. aquifolium).

- Bergamot-extract from Citrus bergamia.

- Soluble fibres-including beta-glucan, glucomannan and psyllium fibre.

- Garlic-extract from Allium sativum.

- Green tea-extract from Camellia sinensis.

- Lupin-lupin proteins.

- Plant sterols and stanols.

- Policosanols.

- Red yeast rice-extracts from Oryza sativa fermented by yeast from the genus Monascus.

- Silymarin-extracts from Silybum marianum.

- Spirulina-microalgae including the species Spirulina platensis, S. maxima and S. fusiformis.

- Soybean-soy proteins. 
- Agents that combine the abovementioned nutraceuticals, for example, combinations of red yeast rice and policosanols, berberine, silymarin or artichoke.

Trials must administer nutraceuticals in the form of a capsule, pill, powder, solution, tablet or enriched food items. We will exclude trials with dietary or exercise co-interventions not applied in the intervention and placebo groups. The use of lipid-lowering drugs in any trial arm will also be a reason for exclusion. Main reason for exclusion of trials comparing nutraceuticals with lipid-lowering drugs is based on the assumption that inclusion of different kind of lipid-lowering drugs would significantly increase heterogeneity between analysed studies. The second reason is that in the authors opinion it would be of little clinical significance, as nutraceuticals should be administered in cases of statin intolerance or non-compliance with prescribed state-of-the-art medical therapy. Therefore, from clinical point of view the aim of this meta-analysis is to answer question which nutraceuticals are the best option in patients in whom nutraceuticals are from various reasons the only available treatment. What is even more important from clinical point of view, until nutraceuticals are proven effective in reducing major cardiovascular events, their comparison with statin would not be desirable, because even in unlikely case of similar LDL-lowering potential one could not deduce on their influence of adverse events.

\section{Outcomes}

Eligible studies are required to report plasma lipid profile results. The primary outcome of this review will be LDL-C. Secondary outcomes will be HDL-C, TC and TG. If available, for purposes of future reports, we will also extract data on plasma non-HDL-C, apolipoproteins (A-I, B, B-100, E), lipoprotein(a) and the appropriate ratios of these markers (eg, LDL-C/HDL-C). The safety profile of nutraceuticals is considered to be high and was extensively characterised by previous reports. ${ }^{14} 24$ Therefore, this review will not summarise data on the safety or adverse effects associated with nutraceutical use.

\section{Study design}

Only randomised controlled trials (RCTs) with either parallel or crossover designs will be included in this systematic review. The study has to compare the efficacy of a nutraceutical with the efficacy of another nutraceutical (head-to-head trials) or the efficacy of a nutraceutical with the efficacy of a placebo. As the Cochrane Handbook suggests, the results of trials presented in the form of conference abstracts or papers will be considered for inclusion if sufficient information is provided. ${ }^{29}$

\section{Search strategy}

The search will be performed by two authors (ML and KO) with any disagreements resolved by discussion with the third author (JM). Three databases, including PubMed, Embase and the Cochrane Central Register of Controlled Trials, will be searched to identify eligible studies. The search will not be limited by date or language of publication. The estimated end date for the searches will be 29 March 2020. Articles in languages other than English or Polish will be translated by a translation service.

An example of the search syntax for PubMed is presented in online supplementary appendix 1. Briefly, the search query will include terms related to the included nutraceuticals and lipid profile parameters. The terms will be searched using free text for the title and abstract fields, as well as a corresponding subject heading (MeSH and Emtree). Moreover, search strategies for PubMed and Embase will be performed with the RCT filters proposed by Cochrane Collaboration. To identify on-going trials, we will additionally search trial registers such as Clinicaltrials.gov (https://clinicaltrials.gov/) and the WHO International Clinical Trial Platform (https://www.who. $\mathrm{int} / \mathrm{ictrp} / \mathrm{en} /$ ). References from included studies, as well as relevant systematic reviews and meta-analyses identified during title/abstract screening, will be checked using Google Scholar for potential eligibility. The results of the database searches will be imported to EndNote X9 software and then automatically and manually examined to remove duplicates.

\section{Selection of studies}

Four authors (TO, KO, ML and GJ) working as pairs of reviewers will independently screen the titles and abstracts of the retrieved articles. Then, full texts of potentially eligible records will be independently screened for eligibility by two reviewers (TO and NP). Any discrepancies during these steps will be resolved by discussion with another author (JM). The results of the study selection, together with reasons for full-text article exclusion, will be presented using a PRISMA-compliant flow diagram. If more than one report from the study is available, the one with a longer follow-up or a larger number of participants will be included.

\section{Data extraction}

A standardised form will be created to extract data for synthesis, risk of bias assessment and identification of potential variables that might modify the effect of the nutraceutical intervention on blood lipids. Data extraction will be performed independently by two authors (KO and ML) with differences resolved by the third author (JM). The following data are planned to be extracted: name of first author, year of publication, study name and country, study design, duration of follow-up, baseline participant characteristics (age, sex, ethnicity, body mass index, disease status), number of arms and allocated subjects, nutraceutical used, dosage, form of administration, postintervention lipid profile (LDL-C, HDL-C, TC, TG and others), number and reason for attrition from study, adverse effects, funding and conflicts of interest.

\section{Risk of bias assessment}

The methodological quality of the studies will be independently assessed by four authors (JM, TO, KO and 
ML) using the Revised Cochrane Risk-of-bias Tool for Randomised Trials (RoB 2) ${ }^{30}$ In contrast to the previous version, the tool is divided into five domains: bias arising from the randomisation process, bias due to deviations from intended interventions, bias due to missing outcome data, bias in the measurement of the outcome and bias in the selection of the reported results. The possible domain-specific and overall risk of bias judgements are a low risk of bias, some concerns and high risk of bias. The overall risk of bias for the study will be ranked as low if all domains are at low risk of bias. If some concerns appear in at least one domain, the judgement on overall risk of bias will increase due to some concerns. In the case of a high risk of bias for one domain or some concerns for multiple domains, which may lower confidence in the results, the risk of bias will be judged as high.

\section{Data analysis}

\section{Measures of treatment effect}

The preferred outcome data will be change scores adjusted for baseline measurements with corresponding $\mathrm{SD}$, followed by post-intervention values and change scores not adjusted for baseline measurements. ${ }^{29}$ In terms of missing $\mathrm{SD}$, we will try to calculate this from the CI, SE, $p$ value or $t$ values if available. If this approach fails, we will use imputation methods, which will then be tested in a sensitivity analysis. ${ }^{29}$ When the study reports the median with the IQR, the mean and $\mathrm{SD}$ will be estimated using the method described by Wan et al. ${ }^{31}$

\section{Standard pairwise and network meta-analyses}

First, for each available comparison of nutraceutical versus control and nutraceutical versus nutraceutical, we will conduct a standard pairwise meta-analysis. Randomeffect models using the DerSimonian-Laire method will be used to pool the estimates. ${ }^{32}$ The pooled results will be presented on forest plots.

Second, in the case of meeting assumptions, a network meta-analysis of included trials will be conducted. The key property of the network meta-analysis (NMA) is the possibility of comparing multiple interventions in a single analysis by combining direct and indirect comparisons. ${ }^{33}$ The results of pooled trials preserve their internal randomisation and account for the correlation between interventions in the case of multiarm trials. The NMA will be performed using a frequentist approach based on a random-effect meta-analysis model. ${ }^{34}$ For the purpose of this analysis, we will assume that all treatment contrasts have the same heterogeneity variance. Relative effect estimates from the NMA will be presented in league tables. Contribution tables will be produced to present the contribution of direct and indirect comparisons to each study contrast.

Another advantage of the NMA is the ability to produce a ranking of treatment effectiveness. We will calculate the P-score, a frequentist analogue of surface under the cumulative ranking curve, for each outcome network. ${ }^{35}$ P-score range from $0 \%$ to $100 \%$, where a higher P-score value indicates that a treatment is more likely to be the best and a lower value indicates that is more likely to be worse.

Analyses will be conducted in R 3.6.0 software (R Foundation for Statistical Computing, Vienna, Austria) using the metafor package for pairwise meta-analyses, as well as the netmeta packages to conduct analyses and visualise the results of the NMA. ${ }^{34} 36$

\section{Network geometry}

The structure of the network, as well as the available direct evidence from the studies, will be presented using network plots. The lumping of nodes will be based on the nutraceutical classification proposed by previous reviews including different substances/groups of substances as a single node. ${ }^{24}$ If a sufficient number of trials is available, we will fit a network including nutraceuticals with different doses or modes of administration as separate nodes.

\section{Assumption of transitivity}

Transitivity assumes homogenous distribution of potential effect modifiers across included trials. ${ }^{33}$ This is a crucial assumption that determines the rationale for conducting the NMA and the validity of its estimates. We will consider the baseline lipid profile, dose of nutraceutical, weight and age of participants as potential effect modifiers.

\section{Assessment of inconsistency}

Inconsistency in a network of interventions means the presence of substantial differences between estimates from the direct and indirect comparisons. ${ }^{33}$ The presence of inconsistency can be examined using both global and local approaches. Locally, the loop-specific approach will be implemented to check for loops of treatments with substantial inconsistency, ${ }^{37}$ and the node-splitting approach will be used to identify a pair of treatment comparisons in which the direct effect differs from the indirect effects in the entire network. ${ }^{38} \mathrm{~A}$ design-bytreatment interaction model and $\mathrm{I}^{2}$ measure of inconsistency testing will be used to assess inconsistency from all connections in the network. ${ }^{39}$

\section{Subgroup and sensitivity analyses}

To explore potential sources of inconsistency and heterogeneity, we will conduct subgroup and meta-regression analyses. Subgroup analysis will be stratified by age, sex, ethnicity, sample size, length of trial follow-up, baseline cholesterol (normocholesterolemic, hypercholesterolemic) and presence of other comorbidities (diabetes, metabolic, syndrome, hypertension). The sensitivity analyses will include (1) studies with low risk of bias and (2) studies using only nutraceuticals in the form of capsules, tablets, drinks or powders.

\section{Detection of small-study effects and publication bias}

'Comparison-adjusted' funnel plots will be used to assess the presence of small-study effects. ${ }^{40}$ Further, contourenhanced funnel plots will be checked to examine 
whether publication bias could be a reason for the smallstudy effect. ${ }^{41}$

\section{GRADING OF RECOMMENDATIONS ASSESSMENT, DEVELOPMENT AND EVALUATION (CERTAINTY OF THE EVIDENCE)}

We will follow the grading of recommendations assessment, development and evaluation (GRADE) approach to rate the certainty of evidence derived from NMA. For each primary outcome, four authors will independently (JM, TO, ML and GJ) rate the certainty of evidence in each of the direct, indirect and network estimates. ${ }^{42}$ Direct estimates will be evaluated with the following GRADE criteria: risk of bias, indirectness, inconsistency and publication bias. As suggested recently by the GRADE working group, consideration of imprecision is not necessary when rating the direct and indirect estimates to inform the rating of NMA estimates. ${ }^{42}$ The indirect estimate assessments will be based on the direct estimate certainty and will be rated down if intransitivity was judged as serious (ie, disease status). The NMA certainty estimates will be based on the direct and indirect estimates certainty (specifically, the higher of the certainty between direct and indirect, was chosen as the certainty of the NMA estimate), and rating down if incoherence or imprecision will be present. ${ }^{42}$

\section{ETHICS AND DISSEMINATION}

This review will summarise findings from primary studies that have received ethical approval. As no individual patient data will be included in our analyses, no further approvals are required. The authors' team consists of researchers with specialisation in the field of cardiovascular medicine, pharmacology and experience in clinical research and systematic reviews. The protocol has been prepared according to the current guidelines and methodology for systematic review network meta-analyses.

In case of any changes in the protocol, details and explanations of these modifications will be described in the final report of this review. The results of this review will be presented at national and international academic conferences as well as published in a peer-reviewed journal with an open access option to reach the widest group of readers.

\section{DISCUSSION}

To the best of our knowledge, this is the first network meta-analysis that summarises current scientific findings on the effects of nutraceuticals on lipid profiles. There are several potential limitations to the findings of this review. First, we will include trials including patients with different comorbidities and LDL-C levels. Moreover, based on the considered interventions, we will merge trials administering nutraceuticals in different forms (both supplements and enriched food items) and doses. Both issues can account for substantial heterogeneity and inconsistency in our network of treatments. However, we aim to solve these with appropriate subgroup analyses. Second, we are aware that there is a limited number of studies comparing different nutraceuticals in headto-head trials, which can produce a sparsely connected network whose findings will be mostly based on indirect trial comparisons.

Our results will extend the recent position paper of the International Lipid Expert Panel. ${ }^{24}$ The clinical decisionmaking process requires the inclusion and comparison of all eligible interventions. ${ }^{33}$ This goal can be achieved by implementing a network meta-analysis to compare different nutraceuticals using both direct and indirect evidence. Furthermore, we will provide a relative ranking of nutraceutical efficiency, which can answer the question of which agent should be considered the most promising in terms of lowering plasma lipids. Our review will be of great interest to international academic societies, national drug agencies, physicians and patients. These findings, together with information regarding their confidence, will provide a basis for formulating evidence-based guidelines for plasma lipid control. Last but not least, we will identify research gaps and the limitations of current trials, which can be addressed in future studies.

\section{Author affiliations}

${ }^{1}$ Department of Cardiology and Internal Diseases, University of Warmia and Mazury in Olsztyn, Olsztyn, Poland

${ }^{2}$ Department of Pharmacology, Faculty of Medical Sciences in Zabrze, Medical University of Silesia, Zabrze, Poland

${ }^{3}$ 2nd Department of Cardiology and Angiology, Silesian Center for Heart Diseases, Zabrze, Poland

${ }^{4} 3$ rd Department of Cardiology, School of Medicine with the Division of Dentistry in Zabrze, Medical University of Silesia, Zabrze, Poland

${ }^{5}$ Institute for Evidence in Medicine, University Medical Center Freiburg, Freiburg, Baden-Württemberg, Germany

${ }^{6}$ Department of Hypertension, WAM University Hospital in Lodz, Medical University of Lodz, Lodz, Poland

${ }^{7}$ Polish Mother's Memorial Hospital Research Institute (PMMHRI), Lodz, Poland ${ }^{8}$ Cardiovascular Research Centre, University of Zielona Gora, Zielona Gora, Poland

Contributors The research question and eligibility criteria were defined by JM, TO, KO, NP, ML, GJ, MG, LS and MB. JM, TO, KO, NP, LS, GJ and MB contributed to the development of the search strategy, data extraction and analysis methods. The manuscript was drafted by JM and T0 and was revised and approved by all authors.

Funding The authors have not declared a specific grant for this research from any funding agency in the public, commercial or not-for-profit sectors.

Competing interests None declared.

Patient and public involvement Patients and/or the public were not involved in the design, or conduct, or reporting, or dissemination plans of this research.

Patient consent for publication Not required.

Provenance and peer review Not commissioned; externally peer reviewed.

Open access This is an open access article distributed in accordance with the Creative Commons Attribution Non Commercial (CC BY-NC 4.0) license, which permits others to distribute, remix, adapt, build upon this work non-commercially, and license their derivative works on different terms, provided the original work is properly cited, appropriate credit is given, any changes made indicated, and the use is non-commercial. See: http://creativecommons.org/licenses/by-nc/4.0/.

\section{ORCID iDs}

Jakub Morze http://orcid.org/0000-0002-7119-0273

Natalia Pawlas http://orcid.org/0000-0002-7551-9371 


\section{REFERENCES}

1 Joseph P, Leong D, McKee M, et al. Reducing the global burden of cardiovascular disease, part 1: the epidemiology and risk factors. Circ Res 2017;121:677-94.

2 Roth GA, Johnson C, Abajobir A, et al. Global, regional, and national burden of cardiovascular diseases for 10 causes, 1990 to 2015. J Am Coll Cardiol 2017;70:1-25.

3 Mozaffarian D, Benjamin EJ, Writing Group Members. Heart disease and stroke Statistics-2016 update: a report from the American heart association. Circulation 2016;133:e38-60.

4 Salami JA, Warraich $\mathrm{H}$, Valero-Elizondo J, et al. National trends in statin use and expenditures in the US adult population from 2002 to 2013: insights from the medical expenditure panel survey. JAMA Cardiol 2017;2:56-65

5 Adhyaru BB, Jacobson TA. Safety and efficacy of statin therapy. Nat Rev Cardiol 2018;15:757-69.

6 Grundy SM, Stone NJ, Bailey AL, et al. 2018 AHA/ACC/AACVPR/ AAPA/ABC/ACPM/ADA/AGS/APhA/ASPC/NLA/PCNA Guideline on the Management of Blood Cholesterol: Executive Summary: A Report of the American College of Cardiology/American Heart Association Task Force on Clinical Practice Guidelines. J Am Coll Cardiol 2019;73:3168-209.

7 Stroes ES, Thompson PD, Corsini A, et al. Statin-associated muscle symptoms: impact on statin therapy-European atherosclerosis Society consensus panel statement on assessment, aetiology and management. Eur Heart J 2015;36:1012-22.

8 Savarese G, De Ferrari GM, Rosano GMC, et al. Safety and efficacy of ezetimibe: a meta-analysis. Int J Cardiol 2015;201:247-52.

9 Kei A, Elisaf MS. Nicotinic acid: clinical considerations. Expert Opin Drug Saf 2012;11:551-64.

10 Lent-Schochet D, Jialal I. Antilipemic agent bile acid Sequestrants. StatPearls. Treasure Island (FL): StatPearls Publishing Copyright (C 2020, StatPearls Publishing LLC, 2020.

11 Marrs JC, Kostoff MD. Discontinuation of statins: what are the risks? Curr Atheroscler Rep 2016;18:41.

12 Ruscica M, Macchi C, Pavanello C, et al. Appropriateness of statin prescription in the elderly. Eur J Intern Med 2018;50:33-40.

13 Aronson JK. Defining 'nutraceuticals': neither nutritious nor pharmaceutical. Br J Clin Pharmacol 2017;83:8-19.

14 Cicero AFG, Colletti A, Bajraktari G, et al. Lipid-Lowering nutraceuticals in clinical practice: position paper from an international lipid expert panel. Nutr Rev 2017;75:731-67.

15 Cicero AFG, Colletti A. Nutraceuticals and blood pressure control: results from clinical trials and meta-analyses. High Blood Press Cardiovasc Prev 2015;22:203-13.

16 Naveen J, Baskaran V. Antidiabetic plant-derived nutraceuticals: a critical review. Eur J Nutr 2018;57:1275-99.

17 Ho HVT, Sievenpiper JL, Zurbau A, et al. The effect of oat $\beta$-glucan on LDL-cholesterol, non-HDL-cholesterol and apoB for CVD risk reduction: a systematic review and meta-analysis of randomisedcontrolled trials. Br J Nutr 2016;116:1369-82.

18 Millán J, Cicero AFG, Torres F, et al. Effects of a nutraceutical combination containing berberine (BRB), policosanol, and red yeast rice $(R y R)$, on lipid profile in hypercholesterolemic patients: a metaanalysis of randomised controlled trials. Clin Investig Arterioscler 2016;28:178-87.

19 Sahebkar A, Pirro M, Banach M, et al. Lipid-Lowering activity of artichoke extracts: a systematic review and meta-analysis. Crit Rev Food Sci Nutr 2018:58:2549-56.

20 Schwingshackl L, Missbach B, Hoffmann G. An umbrella review of garlic intake and risk of cardiovascular disease. Phytomedicine 2016;23:1127-33.
21 Serban M-C, Sahebkar A, Dragan S, et al. A systematic review and meta-analysis of the impact of Spirulina supplementation on plasma lipid concentrations. Clin Nutr 2016;35:842-51.

22 Xiong $X$, Wang $\mathrm{P}$, Li X, et al. The effects of red yeast rice dietary supplement on blood pressure, lipid profile, and C-reactive protein in hypertension: a systematic review. Crit Rev Food Sci Nutr 2017:57:1831-51.

23 Yuan F, Dong H, Fang K, et al. Effects of green tea on lipid metabolism in overweight or obese people: a meta-analysis of randomized controlled trials. Mol Nutr Food Res 2018;62:1601122.

24 Banach M, Patti AM, Giglio RV, et al. The Role of Nutraceuticals in Statin Intolerant Patients. J Am Coll Cardiol 2018;72:96-118.

25 Moher D, Liberati A, Tetzlaff J, et al. Preferred reporting items for systematic reviews and meta-analyses: the PRISMA statement. PLoS Med 2009;6:e1000097.

26 Hutton B, Salanti G, Caldwell DM, et al. The PRISMA extension statement for reporting of systematic reviews incorporating network meta-analyses of health care interventions: checklist and explanations. Ann Intern Med 2015;162:777-84.

27 Moher D, Shamseer L, Clarke M, et al. Preferred reporting items for systematic review and meta-analysis protocols (PRISMA-P) 2015 statement. Syst Rev 2015;4:1.

28 Mannarino MR, Ministrini S, Pirro M. Nutraceuticals for the treatment of hypercholesterolemia. Eur J Intern Med 2014;25:592-9.

29 Higgins JPT, Green S. Cochrane Handbook for Systematic Reviews of Interventions Version 5.1.0 [updated March 2011]. The Cochrane Collaboration, 2011

30 Higgins JPT, Sterne JAC, Savović J, et al. A revised tool for assessing risk of bias in randomized trials. In: Chandler J, McKenzie $\mathrm{J}$, Boutron I, et al, eds. Cochrane database of systematic reviews, 2016.

31 Wan X, Wang W, Liu J, et al. Estimating the sample mean and standard deviation from the sample size, median, range and/or interquartile range. BMC Med Res Methodol 2014;14:135.

32 DerSimonian R, Laird N. Meta-analysis in clinical trials. Control Clin Trials 1986;7:177-88.

33 Riley RD, Jackson D, Salanti G, et al. Multivariate and network metaanalysis of multiple outcomes and multiple treatments: rationale, concepts, and examples. BMJ 2017;358:j3932.

34 Rücker G, Krahn U, König J, et al. netmeta: network meta-analysis using Frequentist methods. R package version 1.0-1., 2019. Available: https://CRAN.R-project.org/package=netmeta

35 Rücker G, Schwarzer G. Ranking treatments in frequentist network meta-analysis works without resampling methods. BMC Med Res Methodol 2015;15:58.

36 Viechtbauer W. Conducting Meta-Analyses in $R$ with the metafor Package. J Stat Softw 2010;36:1-48.

37 Bucher HC, Guyatt GH, Griffith LE, et al. The results of direct and indirect treatment comparisons in meta-analysis of randomized controlled trials. J Clin Epidemiol 1997;50:683-91.

38 Dias S, Welton NJ, Caldwell DM, et al. Checking consistency in mixed treatment comparison meta-analysis. Stat Med 2010;29:932-44.

39 Jackson D, Barrett JK, Rice S, et al. A design-by-treatment interaction model for network meta-analysis with random inconsistency effects. Stat Med 2014;33:3639-54.

40 Chaimani A, Higgins JPT, Mavridis D, et al. Graphical tools for network meta-analysis in STATA. PLoS One 2013;8:e76654.

41 Peters JL, Sutton AJ, Jones DR, et al. Contour-enhanced metaanalysis funnel plots help distinguish publication bias from other causes of asymmetry. J Clin Epidemiol 2008;61:991-6.

42 Brignardello-Petersen R, Bonner A, Alexander PE, et al. Advances in the GRADE approach to rate the certainty in estimates from a network meta-analysis. J Clin Epidemiol 2018;93:36-44. 